16 DO SYMPTOM CONTROL NEEDS DIFFER IN PATIENTS DYING FROM COVID-19? A SPECIALIST PALLIATIVE CARE EXPERIENCE FROM AN ACUTE TRUST

James Renshaw, Dave Dijay, Lisa McAleny, Sarah Cox, Ruth Caulkin. Department of Palliative Care, Chelsea and Westminster Hospital NHS Foundation Trust

\subsection{6/spcare-2021-PCC.34}

Introduction Data around end of life care in COVID-19 patients remains lacking. We present a study of 89 COVID-19 hospital patients who received specialist palliative care (SPC) input, which provides a novel understanding of the symptom needs of those dying from COVID-19.

Methods Retrospective review of health-records of 89 COVID19 patients with SPC involvement between 20/3/20 - 21/4/20. 54 pre-pandemic control patients referred to SPC between 20/ $11 / 19$ - 20/12/19 were included as comparison.

Results and discussion 84\% $(n=75)$ of COVID-19 patients had died at follow-up compared to $44 \%(n=24)$ of controls. Average time from SPC involvement to death was 3.4 days for COVID-19 patients and 8.3 days for controls.

COVID-19 patients were more breathlessness (92\%, $n=69$ vs $75 \%, n=18)$ and pyrexial $(44 \%, n=33$ vs $4 \%, n=1)$ than controls. Control patients were more likely to experience pain $(58 \%, \mathrm{n}=14$ vs $17 \%, \mathrm{n}=13)$. Agitation rates were similar in COVID-19 patients $(45 \%, n=34)$ and controls $(58 \%, n=14)$.

High symptom burden and rapidity of deterioration in COVID-19 patients necessitated an increased use of continuous subcutaneous infusions (CSCI's), (69\%, $\mathrm{n}=52$ vs $46 \%$, $\mathrm{n}=11)$.

Median doses of opioids and benzodiazepines required in CSCI's to control symptoms were similar across both groups. Median $24 \mathrm{hr}$ CSCI morphine equivalent dose of $15 \mathrm{mg}$ $(n=51)$ in COVID-19 patients vs $10 \mathrm{mg}(n=10)$ in controls. Median CSCI midazolam dose of $10 \mathrm{mg}$ in both groups ( $n=40$ vs $n=10$ in controls). Few COVID-19 patients required glycopyrronium $(n=15)$ and antiemetics $(n=5)$ via a CSCI.

Conclusion and recommendations COVID-19 patients have a rapid deterioration and symptomatic dying phase with different symptom profile to control patients. Symptom control in COVID-19 can be achieved with traditional drugs often at low doses, though with earlier use of CSCI's. Education and reassurance for generalists in the early use of opioids and benzodiazepines is essential to managing symptoms of dying COVID-19 patients.

\section{IS ROUTINE INPATIENT COVID-19 TESTING APPROPRIATE IN THE HOSPICE SETTING? AN AUDIT OF CLINICAL PRACTICE DURING THE COVID-19 PANDEMIC}

Anna-Lucia Koerling, Leila Donald. Martlets Hospice

\subsection{6/spcare-2021-PCC.35}

Introduction Nasopharyngeal swabbing for COVID-19 is now standard for hospital admissions. At Martlets Hospice, a paucity of swabs, and reservations about the invasiveness of swabs in patients approaching the end of life, meant that routine inpatient testing was not implemented until June, when a standard operating procedure (SOP) was written. Post-SOP, patients should have been swabbed as soon as possible after admission, unless they were known COVID-19 positive, were actively dying, or were unable to consent. Our audit aimed to ascertain how manageable routine testing is in a palliative care setting.

Methods 142 patients were admitted to Martlets between 1/4/ 2020 and 1/10/2020. $\mathrm{N}=71$ both pre and post SOP implementation. All inpatients' notes were checked for documentation of swab results or why swabbing was inappropriate, and this was checked against the online pathology reporting system ICE.

Results Pre-SOP, 48/71 patients were deemed appropriate to swab. Of the remainder, 16 were known COVID-19 positive, and 7 were actively dying on admission. 44\% of patients deemed appropriate were swabbed, of whom 19\% tested positive for COVID-19. 50\% of those with positive swab results had been asymptomatic, but were swabbed because they had been admitted from high risk hospital wards. Post-SOP, 60/71 patients were deemed appropriate. There were no COVID-19 positive admissions. $70 \%$ of patients deemed appropriate were swabbed, of whom 0\% were COVID-19 positive.

Discussion Our results indicate that routine inpatient testing is feasible and appropriate in hospice settings. Clear guidelines increase the frequency of swabbing and ensure that only appropriate patients are swabbed. Positive swabs in some asymptomatic patients indicate that particularly in the context of a second wave, routine inpatient testing will be necessary, despite the unpleasantness of the procedure. To reach $100 \%$ of appropriate patients tested, integration of our SOP into admission proformas and documentation is being trialled.

\section{RETROSPECTIVE AUDIT TO ASSESS FOR DIFFERENCES BETWEEN PATIENTS DYING OF COVID-19 VS PATIENTS DYING OF OTHER CAUSES}

Jennifer Creed, Kate Tredgett, Lina Alim, Holly Gaze, Sombul Qamar. Gloucestershire Hospitals NHS Foundation Trust

\subsection{6/spcare-2021-PCC.36}

Background Specialist palliative care is essential in caring for patients dying of Covid-19. Facing this new disease, it is essential we develop an understanding of this patient population and how best to manage their end of life. We wanted to assess if there are any significant differences between patients dying of Covid-19 versus those dying of other causes.

Methods We compared the patients who died of Covid-19 in April 2020, with patients who died in April 2020 and April 2019 without Covid-19 who were known to the specialist palliative care team at a district general hospital. We collected the data by retrospective case note review.

Results Total of 39 patients; 12 patients in the Covid-positive group, 15 in the Non-Covid-2020 group and 12 in the NonCovid-2019 group. There were 25 males distributed across the groups, with a comparable median age across the groups. Malignancy was less common in the Covid-positive group but co-morbidities including cardio-vascular disease and type two diabetes were more common. Patients who were Covid-positive; had a shorter window of palliative care team involvement, a lower AKPS at point of referral and were more likely to be in the dying phase of illness. Symptoms of breathlessness were more likely in the Covid-positive group, but they were less likely to have pain. Agitation was present across all groups. The median doses of morphine and midazolam were very similar across all groups and for all groups these treatments were recorded as being effective. 